\title{
Loss to Follow-up Pattern in TB Patients in Rawalpindi, Pakistan
}

MUNAWAR HUSSAIN SOOMRO, ${ }^{1}$ FAI SAL SHAHZAD, ${ }^{2}$ MUHAMMAD AMI R KHAN, ${ }^{3}$ EJ AZ QADEER, ${ }^{4}$ ODD MØRKVE ${ }^{5}$

\begin{abstract}
Background: Tuberculosis is a serious global public health, social and economical problem that affects millions of people particularly in low income countries. The aim of this study was to see the loss to follow up pattern in Tuberculosis patients in district Rawalpindi, Pakistan.
\end{abstract}

Materials and method: A cross-sectional study was conducted in district Rawalpindi between August and October 2010. All sputum smear positive Tuberculosis patients diagnosed and treated in public sector, urban and rural diagnostic centers of the district Rawalpindi during the year 2008 were included in the study.All cases other (smear negative pulmonary and extra-pulmonary) than sputum smear positive and all private hospitals were excluded.

\begin{abstract}
Results: A total of 45 I patients were enrolled in the study. Majority of the patients (89.6\%) were provided with the treatment support. Geographically dropout rate was observed higher in urban patients than in rural $18.7 \%$ vs. $10.5 \%$. On the other hand by patient gender, drop out was seen more in male patients than in females $16.5 \%$ vs. $13.1 \%$. By patients age groups, interestingly patients under the childhood age group all the patients completed the treatment while drop out was observed more in older age group.
\end{abstract}

Conclusions: Loss to follow up pattern was seen more in urban patients, by gender more in male patients and by age groups observed more in older patients.

Key Words: Tuberculosis, Loss to follow up, Rawalpindi, Cross-Sectional Study

\section{Introduction:}

Tuberculosis (TB) is among the top ten causes of global mortality and ranks second among the leading cause of death from infectious disease worldwide, after the human immunedeficiency virus. TB is a serious global public health, social and economical problem that affects millions of people particularly in low income countries. Each year about 9 million people develop TB disease globally and 1.4 million die of this disease. ${ }^{1}$

Directly observed treatment short-course (DOTS) is an internationally recommended treatment strategy for TB patients and for their continuous follow-up during the treatment. ${ }^{2}$ The DOTS, strategy ensures that infectious TB patients are identified and cured using standardized drug combinations. Treatments supporters observe patients as they swallow their drugs daily. Failure to complete treatment poses a significant increase in disease transmission, reactivation and development of drug resistance.

1. Department of Community Medicine, Al-Nafees Medical College, Isra University-Islamabad Campus, Pakistan.

2. Department of Mathematics, University of Bergen, Norway.

3. Association for Social Development, Pakistan.

4. National TB Control Program, Pakistan.

5. Centre for International Health, University of Bergen, Norway. Correspondence : Dr. Munawar Hussain Soomro. Assistant Professor, Department of Community Medicine, Al-Nafees Medical College, Isra University-Islamabad Campus, Pakistan. Email:munawar_soomro@hotmail.com.
The World Health Organization (WHO) declared TB as a global emergency ${ }^{3}$ and National TB Control Program (NTP) Pakistan began implementing the DOTS strategy in 1995, with Baluchistan as pilot province. The Pakistan government declared TB as a national emergency in 2001 and NTP, Pakistan achieved countrywide DOTS coverage in 2005. ${ }^{4}$ Currently Pakistan ranks sixth among the 22 high burden countries of the TB in the world. WHO has set the millennium development goals to control TB with $70 \%$ case detection rate and $85 \%$ cure rate in the DOTS by the year $2015^{5,6}$ The present study was therefore undertaken to see the loss to follow up pattern in TB patients of the district Rawalpindi, Pakistan.

\section{Methodology:}

A cross-sectional study was conducted between August and October 2010 in 15 health facilities of the district Rawalpindi. All sputum smear positive TB patients diagnosed and treated in public sector, urban and rural diagnostic centers during the year 2008 were included in the study. All cases other (smear negative pulmonary and extra-pulmonary) than sputum smear positive and all private hospitals were excluded.

Data was extracted from the patients TB treatment cards (TB01)and TB register (TB03) on a pre-designed form and were entered and analyzed by SPSS/PASWstats version 17.Descriptive statistics were applied, using frequencies and cross tabulations were used to compare group differences for categorical variables. 
Patients were divided into three age groups ${ }^{7}$

Group 1: Age $\leq 14$ yrs (Childhood)

Group 2: Age 15-54 yrs (Productive age)

Group 3: Age $\geq 55$ yrs (Older age)

\section{Results:}

A total of 451 patients were enrolled in the study. Fifty three percent $(n=239)$ of the patients were male. $53.4 \%(n=241)$ of the registered patients lived in urban settings. There were $3.1 \%(\mathrm{n}=14)$ patients in the childhood group, $71.8 \%(\mathrm{n}=$ $324)$ in the productive age group and $25.1 \%(n=113)$ in the older age group. Majority of the patients 89.6\% $(n=404)$ were provided with the treatment support.

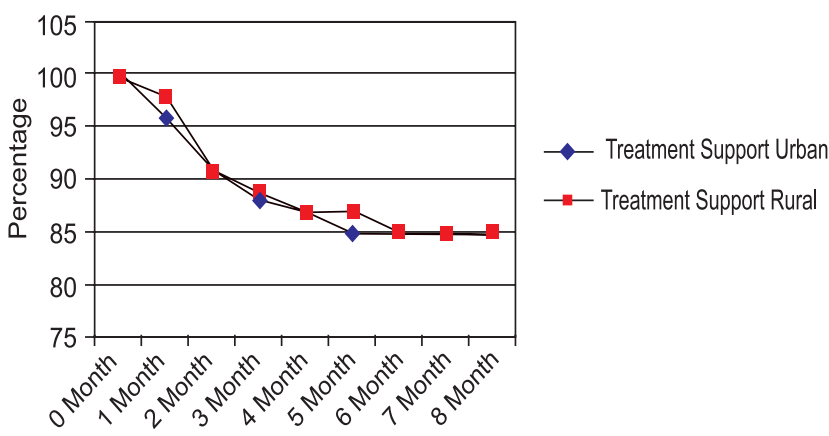

Fig.-1: Loss to follow up pattern in all TB patients

Figure1 shows the loss to follow-up pattern in all TB patients. Among "treatment support", as well as among "no treatment support" $85.1 \%$ of the patient completed the eight months treatment. About $9 \%$ of the patients were dropped out during intensive phase from both groups. Similarly, during continuation phase about $5.9 \%$ of the patients were dropped out in both groups.

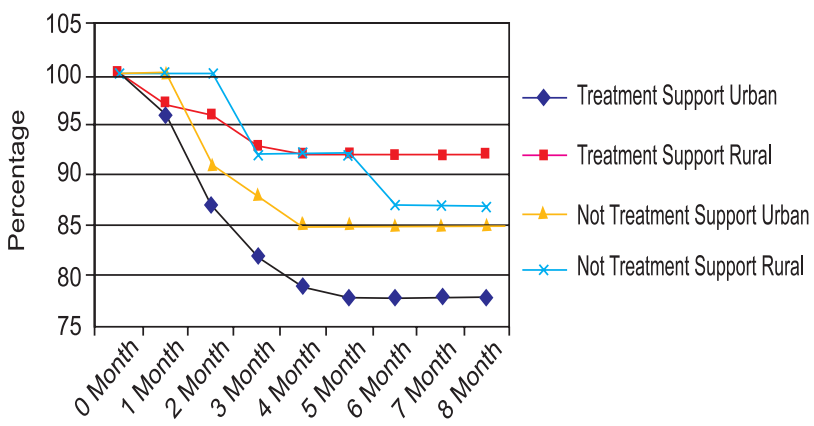

Fig.-2: Loss to follow up pattern by patient geography

Figure2 shows loss to follow-up pattern by patient geography. Among urban patients with "treatment support" $13 \%$ were dropped out during the intensive phase of the treatment while only about 4\% among rural patients with "treatment support". On the other hand those with "no treatment support” 91\% completed intensive phase in urban and $100 \%$ in rural patients. At the end of eight months of treatment results were found better in rural patients with a difference of $8.2 \%$ in drop-out rate between urban and rural patients $18.7 \%$ vs. $10.5 \%$ respectively.

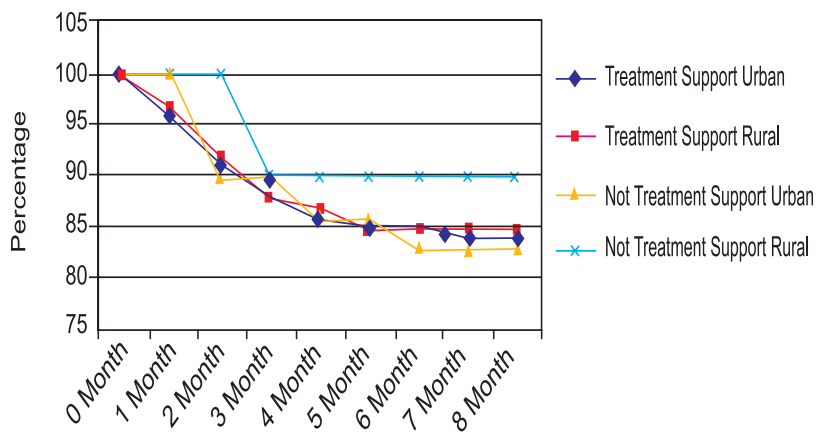

Fig.-3: Loss to follow up pattern by patient gender

Loss to follow-up pattern by patient gender is shown in Figure.3. Among male patients with "treatment support" 9\% were dropped out during the intensive phase of the treatment while only $8 \%$ among female patients with "treatment support". On the other hand those with "no treatment support" 90\% completed intensive phase in male and 100\% in female patients. At the end of eight months of treatment, results were comparatively much better in female than in male patients both with "treatment support" and "no treatment support”. So, by gender results were better in females with a small difference of 3.4\% in drop-out rate between male to female patients $16.5 \%$ vs. $13.1 \%$ respectively.

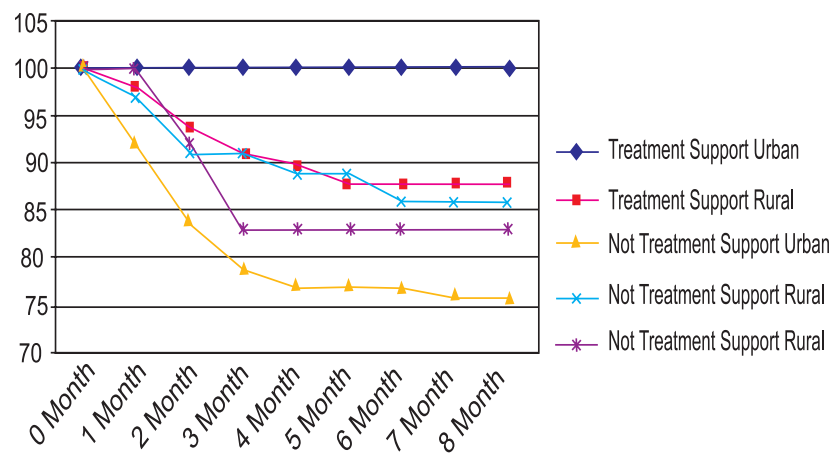

Fig.-4: Loss to follow up pattern by patient age group

Loss to follow-up pattern by patient age group is shown in Figure 4. All the patients with age group d"14 yrs were provided with the treatment support and all completed their intensive phase as well as continuation phase. In patients with age group 15-54 yrs, about 94\% completed their intensive phase in "treatment support" and 91\% in "no treatment support” groups. At the completion of continuation phase 
88\% were among "treatment Support” and 86\% among "no treatment support” group. Among the patients with age group e"55 years, the dropout rate was higher among patients with "treatment support" group (24\%) than the "no treatment support” group (17\%) during intensive phase as well as in continuation phase.

\section{Discussion:}

One of the key findings of our study is that overall $14.9 \%$ of sputum smear positive TB patients were dropped out before completing eight months treatment. The dropout was higher (9\%) during the first two months of treatment (i.e. intensive phase) as compared to that of continuation phase (5.9\%). Similar observations of high initial attrition of TB patients have also been reported from other studies in Pakistan. ${ }^{8,9}$

It is evident that patient adherence to TB treatment is a complex phenomenon, and is affected by multiple factors ranging from characteristics of the individual patient to qualities of social and economic environment. ${ }^{10-14}$ This can be improved by patients counseling, communication and patients' choice of treatment supporter. ${ }^{15-17}$ The regularity of drug intake during intensive phase of treatments seems to predict successful treatment outcomes. The dropout in our study during the intensive phase is contrary to the expectations and indicate relative inability of direct observation alone, to enhance the adherence or reduce dropouts during the first two months of the TB treatment. Defaulting from the treatment is one of the most important problems in TB control globally. Studies have shown that the lack of information, poor communication with health staff, lack of attention and health staff can play a key role in achieving adherence to treatment. ${ }^{15,17-19}$

Another finding of our study was that the dropout rate was seen more in urban than in rural patients. It was also observed that dropout rate was more in male than in female patients. Higher default rate from treatment in males has been documented in the literature. ${ }^{9,19-21}$ One possible explanation to this could be that men face higher opportunity costs of treatment and direct observation because they are more likely to be economically active where as women are likely tobe able to rearrange and share activities tofit with treatment. ${ }^{22}$ Observing patients by their age groups, the dropout rate in older age group patients was higher particularly in the intensive phase than in the continuation phase of the treatment. While in childhood age group patients were found to be $100 \%$ successful in the treatment.Overall we found that treatment success rate was $85.1 \%$ among patients with "treatment support" and "no treatment support", but the number of patients with "no treatment support” was small 10.4\%.
The results of this study showed better treatment success rate. However, there is a strong need to consider implementation of more flexible approaches to direct observation that are responsive to patient's preferences and circumstances. This supplemented by alternate measure to promote adherence can be helpful in achieving the desired levels of treatment outcomes.

\section{Conclusion:}

Loss to follow up pattern was seen slightly more in urban patients, by gender more in male patients and by age groups observed more in older patients'(agee” 55 yrs). Patients' education, encouragement and periodic trainings of the treatment providers can be very helpful in achieving desired levels of treatment outcomes.

\section{Conflict of interest: None}

\section{References:}

1. World Health Organization, Global Tuberculosis Report 2012, WHO/HTM/TB/2012.6. 2012.

2. World Health Organization, Toman's tuberculosis case detection, treatment, and monitoring: questions and answers. WHO/HTM/TB/2004.

3. Arnadottir T., Tuberculosis and Public Health. Policy and Principles in Tuberculosis Control. Paris, France: International Union against Tuberculosis and Lung Disease 2009.

4. TB-Free Pakistan Current Status and the Way Forward: Annual Report 2008, National TB Control Programme, Pakistan 2008.

5. National Tuberculosis Control Programme, Ministry of Health, Government of Pakistan. National guidelines for diagnosis and management of tuberculosis in children 2007.

6. World Health Organization, Treatment of tuberculosis: guidelines - 4th ed. WHO/HTM/TB/2009.420. 2009.

7. Palomino JC, Leao SC, and Ritacco V. Tuberculosis 2007 from basic science to patient care. 2007.

8. MA, Khan. A randomized control trial to assess the effectiveness of direct observation component of the DOTS strategy for tuberculosis, PhD Thesis., 2003, University of Leeds, UK.

9. Liefooghe R, Michiels N, Habib S, Moran MB, De Muynck A. Perception and social consequences of tuberculosis: A focus group study of tuberculosis patients in Sialkot, Pakistan. Social Science \& Medicine 1995; 41(12): 1685-92.

10. Munro SA, Lewin SA, Smith HJ, Engel ME, Fretheim A, Volmink J. Patient Adherence to Tuberculosis Treatment: A Systematic Review of Qualitative Research. PLoS Med 2007; 4(7): 238. 
11. XuWeiguo, Lu wei, Zhou Y, Zhu L, Shen H, Wang J. Adherence to anti-tuberculosis treatment among pulmonary tuberculosis patients: a qualitative and quantitative study. BMC Health Services Research 2009; 9:169.

12. Nissen TN, Rose MV, Kimaro G, Bygbjerg IC, MfinangaSG, Ravn P. Challenges of Loss to Follow-up in Tuberculosis Research. PLoS One 2012; 7(7): 40183.

13. Alcantara CCSD, Kritski AL, Ferreira VG, Facanha MC, Pntes RS, Mota RS, et al. Factors associated with pulmonary tuberculosis among patients seeking medical attention at referral clinics for tuberculosis. J Bras Pneumol 2012; 38(5): 622-29.

14. Obasanjo OO, Shields T, Coggin W, Chaisson RE, Glass GE. The Impact of Directly Observed Therapy for Tuberculosis on Loss to Follow-up in Baltimore City. International Journal of Business and Social Science 2012; 3(2):51-55.

15. Thiam S, LeFevre AM, Hane F, Ndiaye A, Ba F, Fielding $\mathrm{KL}$, et al. Effectiveness of a strategy to improve adherence to tuberculosis treatment in a resource-poor setting: A cluster randomized controlled trial. . JAMA 2007; 297(4): 380-6.

16. Day M, Middlemiss A, and OkerekeTE. What really happens to tuberculosis patients classified as lost to follow-up in West Yorkshire? Euro Surveill 2012; 17(38): 20278.
17. Gust DA, Mosimaneotsile B, Mathebula U, Chingapane B, Gaul Z, Pals SL, et al. Risk Factors for Non-Adherence and Loss to Follow-up in a Three-Year Clinical Trial in Botswana. PLoS One 2011; 6(4):18435.

18. Jaiswal A, Singh V, Ogden JA, Porter JDH, Sharma PP, Sarin $\mathrm{R}$, et al. Adherence to tuberculosis treatment: lessons from the urban setting of Delhi, India. Tropical Medicine and International Health 2003; 8(7): 625-33.

19. Comolet TM, Rakotomalala R, and Rajaonarioa H. Factors determining compliance with tuberculosis treatment in an urban environment, Tamatave, Madagascar. Int J Tuberc Lung Dis 1998; 2: 891-7.

20. Lienhardt C, et al. Factors determining the outcome of treatment of adult smear-positive tuberculosis cases in The Gambia. Int J Tuberc Lung Dis 1998; 2(9):712-8.

21. Connolly C, Davies GR, and W. D. Who fails to complete tuberculosis treatment? Temporal trends and risk factors for treatment interruption in a community-based directly observed therapy programme in a rural district of South Africa. Int J Tuberc Lung Dis1999; 3(12):1081-7.

22. Khan MA, Walley JD, Witter SN, Imran A, Safdar N. Cost and cost-effectiveness of different DOT strategies for the treatment of tuberculosis in Pakistan. Health Policy and Planning 2002; 17(2):178-86. 